\title{
Activity-Dependent Regulation of Tachykinin-Like Immunoreactivity in Neurons of Monkey Visual Cortex
}

\author{
S. H. C. Hendry, ${ }^{1}$ E. G. Jones, ${ }^{1}$ and N. Burstein ${ }^{2}$ \\ Departments of ${ }^{1}$ Anatomy and Neurobiology and ${ }^{2}$ Ophthalmology, University of California, Irvine, California 92717
}

A monoclonal antibody that recognizes the carboxyl terminus of substance $P$ was used to localize tachykinin-like immunoreactivity in neurons of area 17 of the adult monkey cerebral cortex. Tachykinin immunostaining was examined in normal monkeys, in monkeys receiving monocular injections of the sodium channel blocker TTX for 10 or $15 \mathrm{~d}$, and in monkeys from which the crystalline lens of one eye had been removed 3 or 6 months prior to death. The immunocytochemical staining in each monkey was compared with the histochemical staining for the mitochondrial enzyme cytochrome oxidase (CO). These forms of monocular deprivation produce the most profound changes in the staining of layers II-III and IVC.

In layers II-III of normal monkeys, tachykinin-immunoreactive somata are uniformly distributed but immunostained puncta are densely packed in rows of patches that correspond to the rows of CO-stained patches. Following monocular TTX injections, both the patches of CO staining in the deprived-eye columns and the corresponding patches of intense tachykinin immunostaining shrink. Quantitative analyses indicate the numerical density of immunostained somata is reduced by $50 \%$ within the deprived-eye rows of patches and is also reduced within regions surrounding the patches in both sets of ocular dominance columns. Following the removal of the lens from one eye, the CO-stained patches and the immunostained patches in one set of rows shrink and the density of immunostained somata in these rows is reduced by $60 \%$. In the alternating rows, the CO staining between patches increases so that many of the patches fuse to form long, continuous bands. Patches of immunostained puncta also enlarge and fuse; the density of immunostained somata in these rows of enlarged patches is approximately $30 \%$ greater than normal.

In layer IVC of normal monkeys, the $\mathrm{CO}$ staining and the tachykinin immunostaining are relatively uniform. Following monocular TTX injections the CO staining and the tachykinin immunostaining are greatly reduced in columns dominated by the injected eye, corresponding to an $80 \%$ reduction in the numerical density of immunoreactive somata. By contrast, the CO staining in layer IVC of aphakic monkeys is changed only slightly from normal and the tachykinin im-

\footnotetext{
Received Apr. 7, 1987; revised July 29, 1987; accepted Sept. 10, 1987.

Supported by Grants EY 06432, EY 07193, and NS 21377 from the National Institutes of Health, United States Public Health Service. S.H.C.H. is an Alfred P. Sloan Foundation Fellow.

Correspondence should be addressed to Dr. Edward G. Jones at the above address.

Copyright (C) 1988 Society for Neuroscience $0270-6474 / 88 / 041225-14 \$ 02.00 / 0$
}

munostaining appears normal. The changes in the density of immunostained somata in both layers II-III and in IVC occur even though the total density of thionin-stained neurons remains normal.

These data suggest that tachykinin-like immunoreactivity in area 17 neurons is activity-dependent: less activity reduces the concentration of tachykinin-like substances in cortical neurons, making them undetectable by immunocytochemistry, while greater activity increases the concentration of these substances, leading to a greater number of cells stained immunocytochemically. This activity-dependent control of tachykinins may contribute to functional changes in the adult visual cortex that occur following monocular deprivation or enucleation.

Monocular deprivation during a critical period of postnatal development leads to dramatic changes in the connectivity and functional properties of neurons in the monkey visual cortex (area 17; Hubel et al., 1977; Blakemore et al., 1978; von Noorden, 1985). Most commonly, these changes are seen physiologically and anatomically as the expansion of cortical columns dominated by the intact eye into regions that would normally become dominated by the deprived eye. By contrast, monocular deprivation or even removal of an eye in the adult monkey leads to no detectable changes in cortical anatomy (LeVay et al., 1980). Still, functional expansion may occur in monocularly enucleated monkeys, so that columns driven by the intact eye are abnormally wide, while zones unresponsive to visual stimuli are extremely narrow (LeVay et al., 1980). The basis for such a plastic response is not known.

Neurons in the PNS respond to changes in their activity not only by acquiring new synaptic contacts or stabilizing existing contacts, but also by altering the synthesis of neurotransmitters and their receptors and synthesizing enzymes and other molecules mediating the actions of second messengers. For example, in cells of the superior cervical ganglion, the synthesis of substance $\mathrm{P}$ (SP) is reduced and the synthesis of norepinephrine increased after receiving depolarizing stimuli that increase neuronal activity (Black et al., 1984). Increased synthesis of SP and reduced synthesis of enkephalin result from reduced activity in presynaptic fibers innervating cells of the adrenal medulla. Similar kinds of regulation of neurotransmitter (Baker et al., 1983; Kantner et al., 1985) and receptor (LaMotte et al., 1976; Ninkovic et al., 1981) expression have also been reported for some central neurons.

Recently, monocular deprivation in adult monkeys has been shown to reduce the immunocytochemical staining for the neurotransmitter GABA and its synthesizing enzyme glutamate decarboxylase (GAD) in neurons of deprived-eye dominance col- 
umns in area 17 of the monkey visual cortex (Hendry and Jones, 1986). This finding suggests that the synthesis of GAD and GABA is regulated by the levels of visually evoked activity reaching cortical neurons. The preceding paper (Jones et al., 1988) demonstrates that a significant proportion of GABA-immunoreactive neurons in monkey cerebral cortex also display tachykinin-like immunoreactivity. In area 17, the GABA-/ tachykinin-positive cells are most numerous in layer IVC (Jones et al., 1988), where the clearest activity-dependent regulation of neuronal GAD and GABA immunoreactivity is found (Hendry and Jones, 1986). Does activity also regulate the levels of tachykinin-like immunoreactivity in these cells and in other neurons of monkey visual cortex? In the present study we used manipulations of one eye to decrease and increase the apparent levels of metabolic activity in monkey visual cortical neurons and found that the levels of tachykinin-like immunoreactivity follow precisely the changes in activity.

\section{Materials and Methods}

Nine monkeys (Macaca fascicularis) were used in this study. Two were normal, 3 received intraocular injections of TTX $(15 \mu \mathrm{g}$ in $10 \mu \mathrm{l}$ of normal saline) every fifth day for 10 or $15 \mathrm{~d}$ before death and 4 had the crystalline lens of one eye surgically removed 3 months or 6 months before death. The interval between intraocular TTX injections was somewhat longer than that used in a previous study of the cat visual system (Stryker and Harris, 1986), but examination of direct and consensual pupillary light reflexes indicated that retinal activity remained suppressed throughout the $5 \mathrm{~d}$ period. All animals were killed with an overdose of Nembutal, followed by perfusion through the heart with $2 \%$ paraformaldehyde and $0.1-0.2 \%$ glutaraldehyde in $0.1 \mathrm{M}$ phosphate buffer. The occipital lobes from each monkey were cut into one narrow (5-mm-wide), sagittally oriented block through area 17 and one or 2 larger blocks that included the remainder of area 17. The larger blocks were flattened and all blocks were frozen after being sunk in a $30 \%$ sucrose/phosphate buffer solution at $4^{\circ} \mathrm{C}$. Sections from the flattened blocks were cut parallel to the opercular surface of the occipital lobe, and those from the narrow block were cut sagittally. Sections from both types of blocks were cut serially, alternately at 20 and $40 \mu \mathrm{m}$. The thicker sections were reacted histochemically for cytochrome oxidase $(\mathrm{CO}$; WongRiley, 1979) and the thinner were either processed for tachykinin-like immunoreactivity, using the monoclonal antibody NC1/34HL (Sera Labs; diluted 1:500 or 1:1000), as described in the preceding paper (Jones et al., 1988) or stained with thionin. Both eyes of each monkey were examined histologically and the dorsal lateral geniculate nuclei (LGN) of each monkey were stained for $\mathrm{CO}$ and with thionin. In addition, $2 \mathrm{~d}$ before the animal was killed, the eyes injected with TTX were also injected with a mixture of $10 \mu \mathrm{l}$ of ${ }^{3} \mathrm{H}-\mathrm{L}$-proline and ${ }^{3} \mathrm{H}-\mathrm{L}-$ leucine (sp act of $21.8 \mathrm{Ci} / \mathrm{mmol}$ and $32.4 \mathrm{Ci} / \mathrm{mmol}$, respectively; final activity, $25 \mu \mathrm{Ci} / \mu \mathrm{l})$ and $10 \% \mathrm{HRP}$. Twenty-micron-thick sections through each LGN were mounted on glass slides and processed for autoradiography as described elsewhere (Jones et al., 1979) or were reacted in a free-floating way for peroxidase histochemistry (DeOlmos et al., 1978).

Quantitative analyses. Three types of quantitative analyses were performed:

1. The numerical density of tachykinin-immunoreactive somata was calculated for patches coinciding with CO-stained patches in layers II and III, for the regions between patches within a row of patches (interpatch), and for the regions between rows of patches (inter-row). These values were determined for normal, TTX-injected, and aphakic monkeys. The patches and the inter-patch and inter-row regions were identified in CO-stained sections and were drawn at $500 \times$ magnification on a sheet of paper by the use of a camera lucida. Landmarks, such as blood vessels were included in the drawing. The adjacent, immunocytochemically stained section was then placed under the microscope and the drawing superimposed over the appropriate region by aligning the landmarks. Immunostained somata were identified and marked on the drawing. The number of somata was determined; the areas of the patches and of the inter-patch and inter-row regions and the immunostained cell density within each were calculated with a Zeiss Zidas digitizing tablet. Thirty patches and inter-patch and inter-row regions were examined in each of 3 sections from each monkey. Numerical densities were determined separately for patch, inter-patch and inter-row regions from the 2 sets of ocular dominance columns in each monkey. For the monocularly deprived monkeys, each set of columns could be identified by the differences in their $\mathrm{CO}$ staining. For the normal monkeys, the values for different columns were determined by calculating the density for patches, inter-patch, and inter-row regions in every other row of patches and by collecting these valucs separately from the values for the intervening rows of patches. The density within inter-row regions was determined for each set of columns by restricting the analyses to regions within $100 \mu \mathrm{m}$ of borders with the adjacent rows of patches.

2 . The numerical density of tachykinin-immunoreactive neurons and thionin-stained neurons was calculated for layer IVC of normal monkeys, and for darkly stained and lightly stained CO bands in TTXinjected monkeys. One hundred-micron-wide traverses through the thickness of layer IVC were marked out with a calibrated rectangle that was superimposed onto the sections through a camera lucida. The number of immunostained somata was determined for 30 traverses through layer IVC of each normal monkey. Darkly stained and lightly stained bands were identified in CO-stained sections and, by the method described above, were superimposed onto adjacent immunocytochemically stained sections. The number of somata in 30 darkly stained and 30 lightly stained bands was determined for each TTX-injected monkey.

3. The number of tachykinin-immunoreactive neurons was determined for rows of CO patches $100 \mu \mathrm{m}$ wide and $3 \mathrm{~mm}$ long in layers II-III. Normal, TTX-injected, and monocularly aphakic monkeys were examined. The 3-mm-long rows were drawn onto paper at $100 \times$, with care being taken to keep the $\mathrm{CO}$ patches at the centers of the curved rows by dividing the row into a series of straight-line segments. Starting points were picked arbitrarily and end points were determined to yield a total length of $3 \mathrm{~mm}$. Landmarks were included on the drawing to guide the subsequent counting from immunocytochemically stained sections. The number of immunostained cell bodies was determined for 30 rows in each normal monkey. Thirty rows were also counted for each experimental monkey -15 for columns dominated by one eye, and 15 for columns dominated by the other. The procedures outlined abovc were used on each monkey to determine values for patches, inter-patch regions, and inter-row regions in both sets of ocular dominance columns.

Statistical analyses of the quantitative data were performed using ANOVA or Student's $t$ test. The analyses were done using the mean value for each monkey and for each measure (patch, inter-patch, and inter-row). Sample sizes were calculated for each measure to give realistic estimates of the total population by determining means and standard deviations for subsets of various sizes. Standard deviations initially decreased as a function of sample size and then leveled out. Final sample sizes were larger than the size of the sample subsets necessary to reach a constant standard deviation.

\section{Results}

\section{Normal monkeys}

In sagittal sections cut radially through area 17 of a normal monkey, both $\mathrm{CO}$ staining and tachykinin immunostaining are densest in 2 layers, IVA and IVC (Fig. 1). In these layers, a high density of immunoreactive somata and punctate profiles is present, the somata belonging to the population of small, relatively lightly stained cells (Jones et al., 1988). The distribution of the immunostained elements (Fig. $1 A$ ) and of CO staining (Fig. 1B) in layers IVA and IVC appears uniform in normal monkeys. A high density of small tachykinin-immunoreactive somata and punctate profiles is also found in layers II and III (Jones et al., 1988), but, like the CO staining in these layers, the immunostaining is uneven. Patches of tachykinin immunostaining, approximately $100-150 \mu \mathrm{m}$ in diameter, extend through layers II and III and are separated from one another by wider regions (350-500 $\mu \mathrm{m}$ wide) that appear more lightly stained (Fig. 1A). The same type of spacing is seen for $\mathrm{CO}$ staining in adjacent sections (Fig. 1B). These tachykinin-stained patches are seen at high magnification to contain the same density of immunoreactive somata as the surrounding regions (see below), but to include a greater density of immunostained punctate profiles.

In tangential sections through layers II and III of area 17 , the 

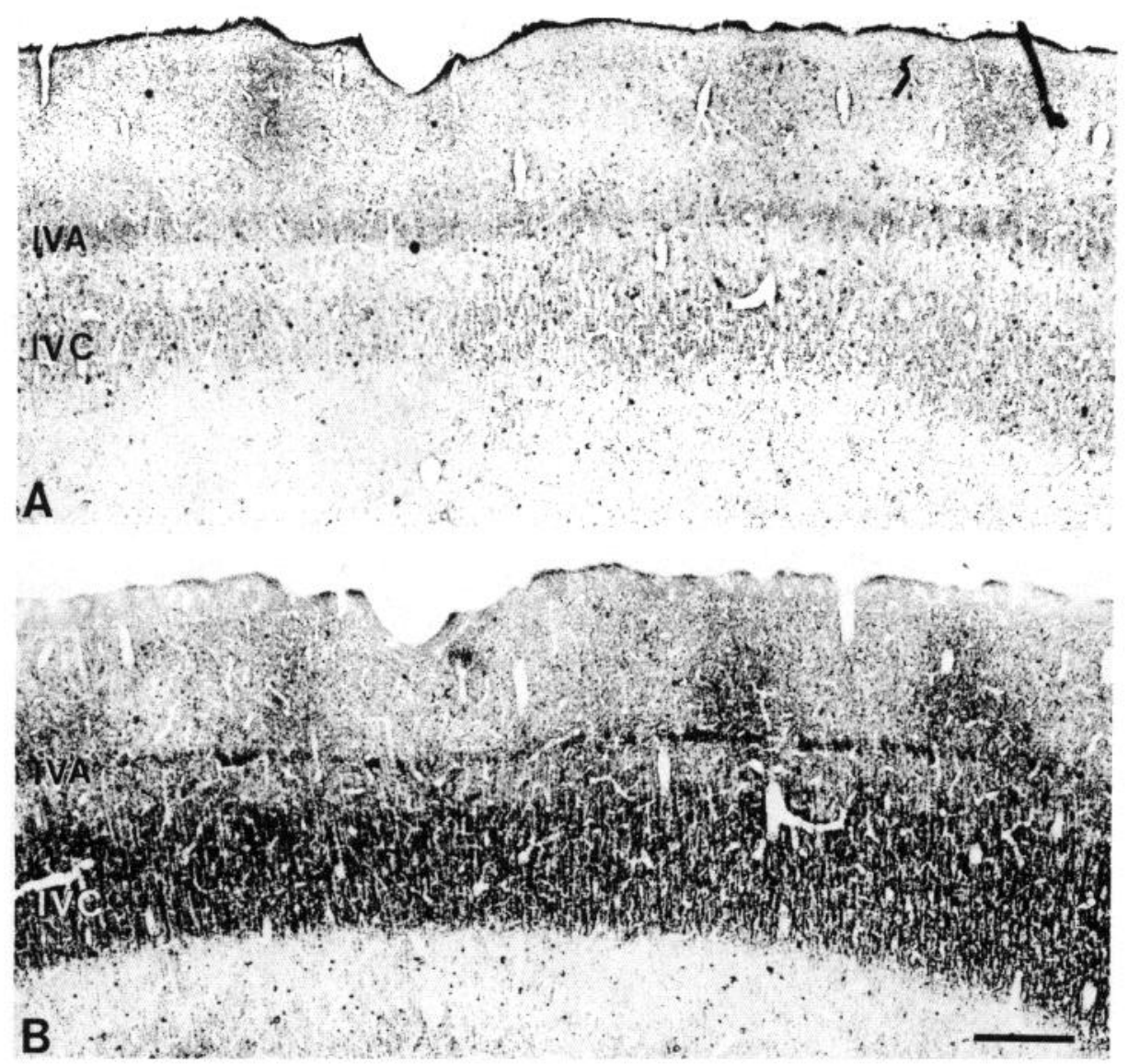

Figure 1. Tachykinin immunostaining $(A)$ and $\mathrm{CO}$ staining $(B)$ in sagittal sections through area 17 of a normal monkey. Both tachykinin-like immunoreactivity and $\mathrm{CO}$ staining are densest in 2 horizontal laminae that correspond to layers IVA and IVC. The staining in these layers appears homogeneous. In addition, patches of tachykinin immunostaining and $\mathrm{CO}$ staining are present in layers II and III. Bar, 200 $\mu \mathrm{m}$. darkly stained $\mathrm{CO}$ patches line up in rows (Fig. $2 B$ ) that lie at the center of ocular dominance columns (Horton and Hubel, 1981; Humphrey and Hendrickson, 1983). Most patches are discrete regions of intense staining, $120-200 \mu \mathrm{m}$ in length, that are separated from adjacent patches within a row by narrow (50-100 $\mu \mathrm{m}$ long), lightly stained regions. However, the staining between some patches is relatively dark, so that within a row 2 adjacent patches can appear to fuse (Fig. $2 B$ ). Similar patches are present in layers V and VI and in one subdivision of layer IV (layer IVB). In the other subdivisions of layer IV, the CO staining forms a dark, honeycomb pattern in layer IVA and a uniformly dark lamina in layer IVC (Fig. $2 B$ ).

In the tangential sections, the distribution of tachykinin-positive cell bodies in layers II-III is relatively homogeneous, but dense clusters of immunoreactive punctate profiles form patches that are the same size and are spaced the same as the CO-stained patches. Each patch contains 10-15 small, lightly stained somata that are embedded in a dense plexus of punctate profiles. The same density of somata exists between the patches (Fig. 3), but the density of puncta is much lower than that seen within the patches. When the positions of the CO-stained patches and the tachykinin-positive patches are superimposed from adjacent sections, the 2 are found to coincide precisely. Each $\mathrm{CO}$ patch lines up with a patch of immunostained punctate profiles, and the $\mathrm{CO}$ and tachykinin-positive patches form superimposable rows (Fig. 2, $A, B$ ). Scattered equally within and between the immunostained patches are a few larger, intensely stained somata.
The numerical density of immunostained cell bodies within the CO-stained patches was compared with that of cells in regions that separate the patches, both within a row (inter-patch) and between rows (inter-row). These analyses show that no statistically significant difference exists between the numerical densities of tachykinin-immunoreactive somata within patches, inter-patch regions, and inter-row regions (Fig. 3).

In sections through layers II-III processed with primary antibody that had been preadsorbed with an excess of synthetic SP, substance K (SK), or neuromedin K, all staining of cell bodies, processes, and punctate profiles is abolished. What remains is a light, diffuse staining in which no periodicities are apparent (Fig. $2 \mathrm{C}$ ), in contrast to the $\mathrm{CO}$-stained patches in the adjacent section (Fig. 2D).

Layers IVA and IVC contain numerous small, tachykininimmunoreactive somata and punctate profiles that, in tangential sections, are uniformly distributed (Fig. 4A). No bands or other periodicities are apparent in the immunocytochemically stained sections (Fig. $4 A$ ) or the adjacent CO-stained sections (Fig. $4 B$ ). The concentration of immunostained elements in layers IVA and IVC is equal to the concentration within the layer II-III patches. In layers IVB, V, and VI, very few of the small, lightly stained somata are present, and in tangential sections through these layers the cells form no detectable pattern. A relatively large number of the intensely stained somata and processes, described in the preceding paper (Jones et al., 1988), is present in layers V and VI, but their distribution within these layers appears uniform. 

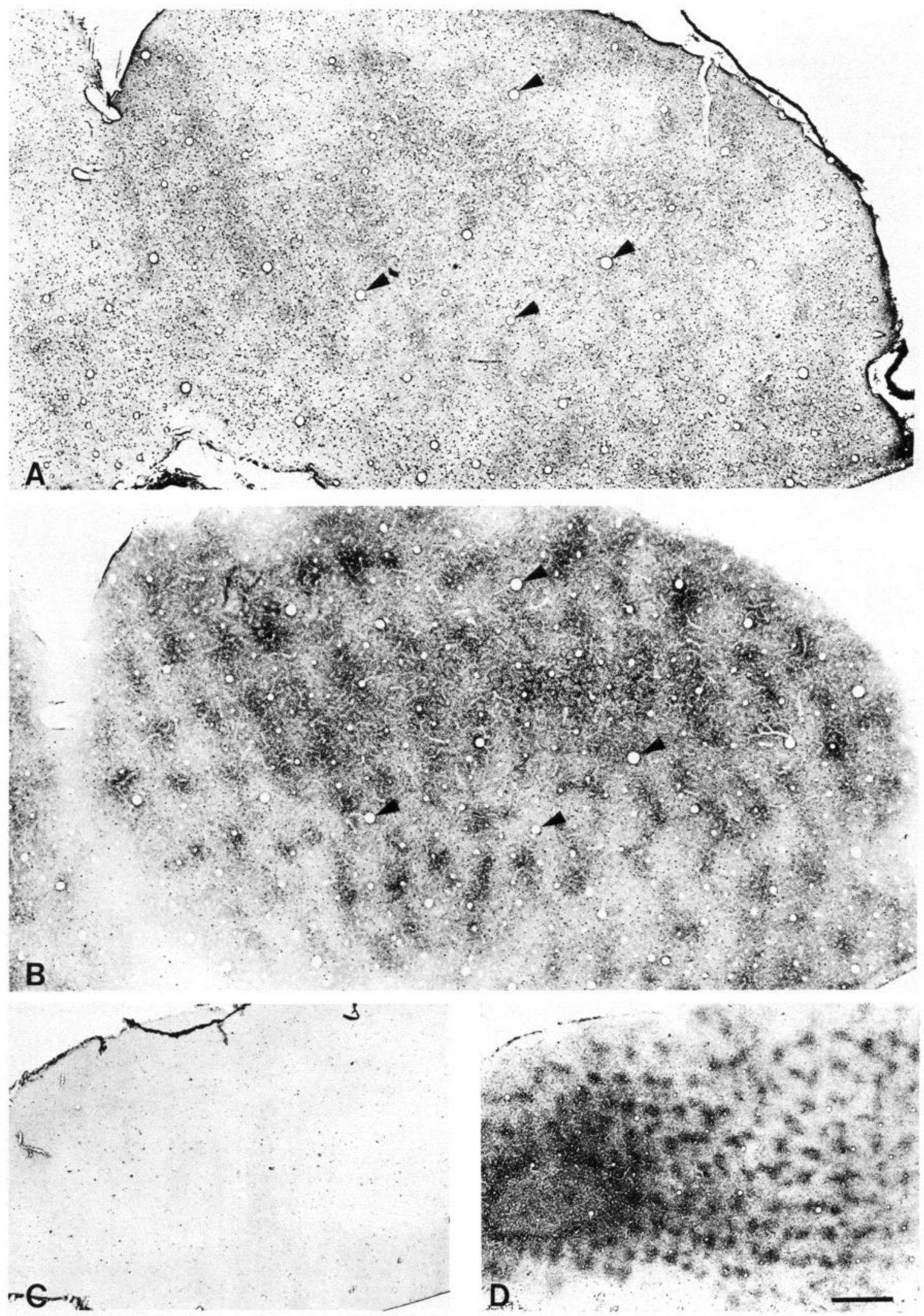

Figure 2. Comparison of immunocytochemical staining $(A, C)$ with $C O$ staining $(B, D)$ in tangential sections through area 17 of normal monkeys that include layers I-III. $A$, Patches of intense tachykinin immunostaining form discontinuous rows in layers II and III. The patches are made up of dense clusters of punctate profiles. $B$, Rows of CO-stained patches are present in layers II and III in a section adjacent to the one shown in $A$. Comparison of the positions of the same blood vessel profiles in $A$ and $B$ (arrowheads) reveals that patches of intense immunostaining in $A$ coincide with patches of intense $\mathrm{CO}$ staining in $B$. $C$, Control immunostaining that results from using primary antibody preadsorbed with an excess of synthetic substance P. The light, diffuse staining is interrupted only by fragments of pia mater and red blood cells that are stained nonspecifically. There is no periodic, patchy staining such as that seen in $A . D$, CO staining of the section adjacent to the one shown in $C$. Rows of intensely stained patches are also present in this section. $\operatorname{Bar}, 1 \mathrm{~mm}(A, B) ; 2 \mathrm{~mm}(C, D)$. 


\section{Monocular injections of TTX}

Following 10 or $15 \mathrm{~d}$ of TTX injections into one eye of an adult monkey, dramatic changes occur in the pattern of $\mathrm{CO}$ staining in the LGN and in area 17 (Fig. 5A; Wong-Riley and Carroll, 1984). Layers of the LGN receiving inputs from the injected eye are stained very lightly (Fig. $5 A$ ), even though the retinal axons projecting to these layers are shown to be intact by their capacity to transport HRP and radiolabeled macromolecules to their terminations (Fig. 5B). In sagittal sections through area 17 of the TTX-injected monkeys, the CO staining in layers IVA and IVC is composed of alternating light and dark bands, corresponding to ocular dominance columns for the injected and uninjected eyes, respectively (Fig. 6A).

The distribution of cells displaying tachykinin-like immunoreactivity in layers IVA and IVC also changes following TTX injections. Immunostained cell bodies and processes are not distributed uniformly, as in normal monkeys, but are organized into bands containing many tachykinin-positive neurons that alternate with bands containing few stained cells (Fig. $6 B$ ). When the tachykinin immunostaining in layer IVC is compared with the $\mathrm{CO}$ staining, the 2 patterns are found to be identical: dark $\mathrm{CO}$ bands coincide with intensely stained tachykinin bands and light $\mathrm{CO}$ bands with lightly stained tachykinin bands.

In tangential sections through area 17 of the TTX-injected monkeys, the CO staining in layers II and III consists of rows of normal patches that alternate with rows of shrunken patches (Fig. 7, B, D). The normal rows overlie ocular dominance stripes in layers IVA and IVC that are darkly stained, while the rows of shrunken patches overlie stripes that are lightly stained. Tachykinin immunostaining in layers II-III closely resembles the $\mathrm{CO}$ staining in these layers. Rows of patches that appear normal alternate with rows in which the patches of immunostained somata and punctate profiles are shrunken (Fig. $7 A$ ). These coincide with the normal and shrunken $\mathrm{CO}$-stained rows of patches seen in adjacent sections (Fig. $7 B$ ). In layer IVC of the TTX-injected monkeys, the distribution of tachykinin-immunoreactive elements is no longer uniform, but is divided into alternating darkly and lightly stained bands (Fig. 7, $A, C$ ) that correspond to the darkly stained and lightly stained $\mathrm{CO}$ bands (Fig. 7, B, D). At high magnification, the density of immunostained somata and puncta is found to be greatly reduced within the light bands (Fig. $7 E$ ), with only a few of the lightly stained

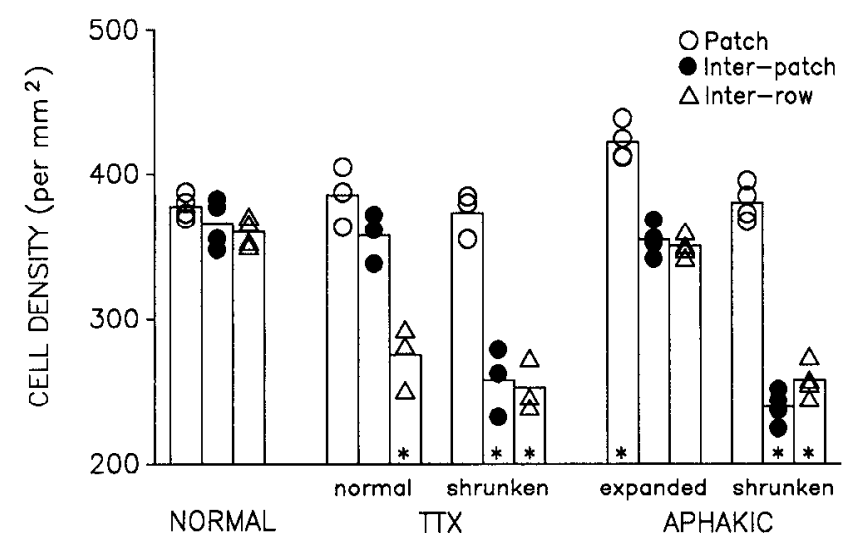

Figure 3. Bar graph of the density of tachykinin-immunoreactive neurons in $\mathrm{CO}$ patches, in regions between $\mathrm{CO}$ patches within a row of patches (inter-patch), and in regions between rows of $\mathrm{CO}$ patches (interrow). Densities were determined for normal, TTX-injected, and aphakic monkeys. Asterisks designate the values that differ from values in normal monkeys at a significance of $p<0.05$ or less. For each normal monkey, 2 values were determined in the 3 regions, representing densities within alternating rows of $\mathrm{CO}$ patches (see Materials and Methods).

type of somata evident over considerable lengths in a band. The numbers of somata and puncta within the darkly stained bands appear normal. These differences between bands are present in sections reacted with either dilution (1:500 or 1:1000) of primary antibody.

Using the $\mathrm{CO}$ staining of adjacent sections as a guide, we counted the number of tachykinin-positive somata in $100 \mu \mathrm{m}$ wide traverses through the thickness of layer IVC in sagittal sections of normal and TTX-injected monkeys. The numbers of somata from lightly stained and darkly stained bands of the injected monkeys were compared with one another and with the numbers from similar counts through layer IVC of normal monkeys that were processed identically. The number of immunoreactive cell bodies per $100-\mu \mathrm{m}$-wide traverse within the darkly stained $\mathrm{CO}$ bands did not differ significantly from the number in layer IVC of normal monkeys, but did differ significantly from the number in lightly stained $\mathrm{CO}$ bands $(t=13.2$, $p<0.0001$; Table 1). These numerical densities did not change with changes in the concentration of primary antibody. The total

Table 1. Number of tachykinin-immunoreactive and thionin-stained somata in 100- $\mu \mathrm{m}$-wide traverses through the thickness of layer IVC

\begin{tabular}{|c|c|c|c|c|c|}
\hline \multirow[b]{2}{*}{ Type of row } & & \multicolumn{2}{|c|}{ Tachykinin } & \multicolumn{2}{|l|}{ Thionin } \\
\hline & & $\overline{\text { Range }}$ & Mean $\pm \mathrm{SD}$ & $\overline{\text { Range }}$ & Mean \pm SD \\
\hline \multirow[t]{2}{*}{ Normal } & (1) & $9-16$ & $13.4 \pm 2.2$ & $138-169$ & $152.6 \pm 11.8$ \\
\hline & (2) & $7-17$ & $12.7 \pm 3.9$ & $134-171$ & $148.2 \pm 12.0$ \\
\hline \multirow[t]{3}{*}{ TTX (normal) } & (1) & $6-16$ & $11.2 \pm 3.8$ & $130-166$ & $147.0 \pm 14.2$ \\
\hline & (2) & $10-17$ & $13.7 \pm 2.6$ & $142-170$ & $154.6 \pm 8.4$ \\
\hline & (3) & $8-17$ & $13.1 \pm 2.2$ & $136-165$ & $151.2 \pm 10.1$ \\
\hline \multirow[t]{3}{*}{ TTX (pale) } & (1) & $0-5$ & $2.1 \pm 1.4$ & $141-173$ & $156.3 \pm 11.2$ \\
\hline & (2) & $0-7$ & $2.6 \pm 1.9$ & $132-166$ & $157.8 \pm 13.2$ \\
\hline & (3) & $0-5$ & $2.6 \pm 2.0$ & $132-171$ & $150.5 \pm 10.3$ \\
\hline
\end{tabular}



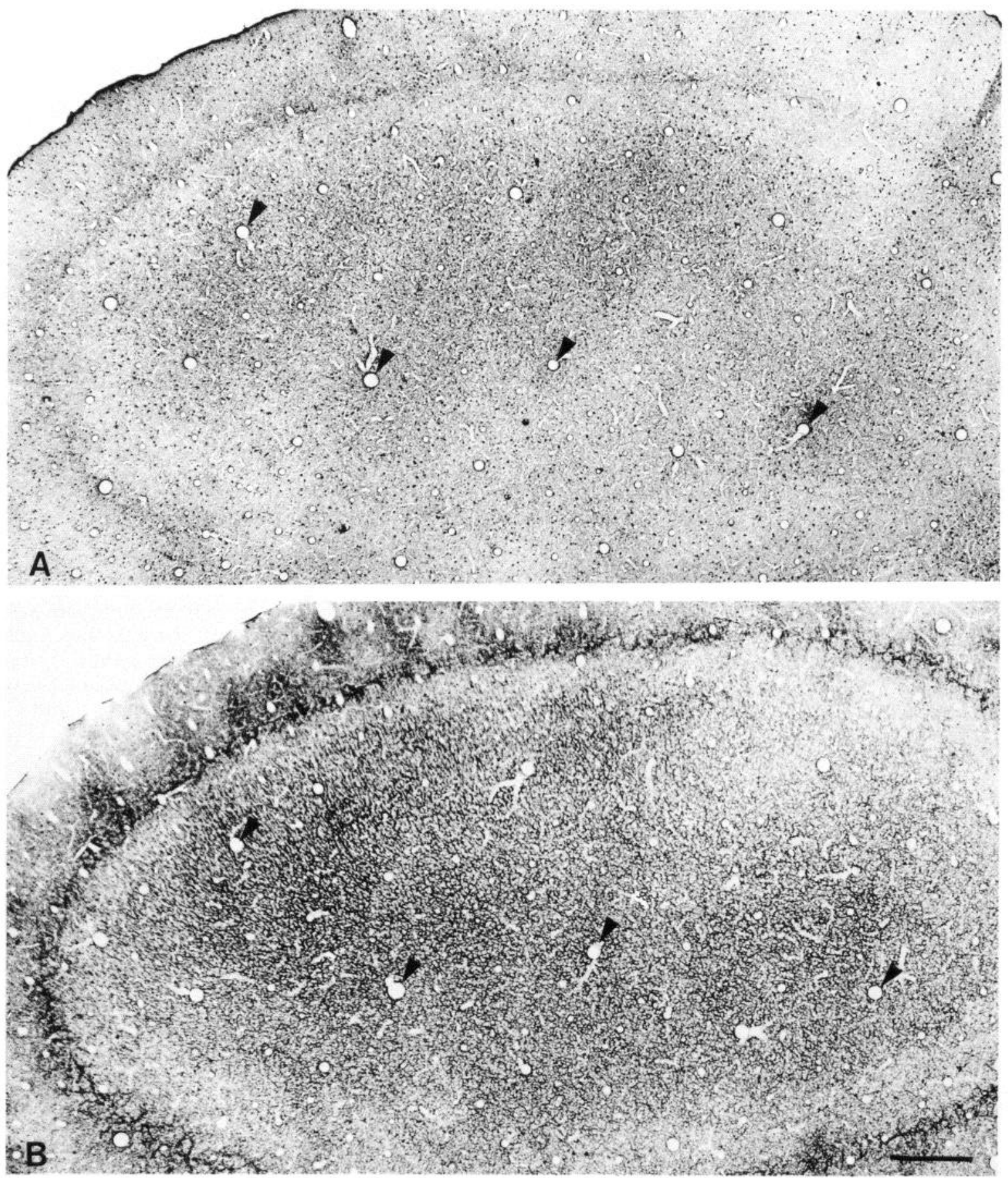

Figure 4. Photomicrographs of tachykinin immunostaining $(A)$ and $\mathrm{CO}$ staining $(B)$ in tangential sections through layers I-IVC of a normal monkey. The central, oval region in each micrograph corresponds to layer IVC. Both the tachykinin immunostaining and the CO staining in layer IVC are intense and relatively homogeneous. Although some variability in the density of immunostained elements is seen in $A$, the changes in staining are found over wide distances and are not periodic. A similar lack of periodicity is also seen in CO-stained sections $(B)$. Bar, $500 \mu \mathrm{m}$.

number of thionin-stained neurons in similar traverses through layer IVC did not differ among normal monkeys, dark bands of TTX-injected monkeys, or light bands of TTX-injected monkeys (Table 1).

The changes in immunostaining within layers II and III were also quantified by examining strips $3 \mathrm{~mm}$ long and $100 \mu \mathrm{m}$ wide, entirely confined to rows of shrunken (CO-stained or immunostained) patches or to rows of normal patches. The number of tachykinin-immunoreactive somata in the rows of normal patches is almost $50 \%$ greater than the number in the rows of shrunken patches (Table 2 ). This difference is statistically significant $(t=10.9, p<0.001)$. However, the difference between rows does not result from differences in the numerical density of immunostained cells within individual patches; i.e. the density of tachykinin-immunoreactive somata within normal patches does not differ significantly from the density within shrunken patches (Fig. 3). Instead, the inter-patch regions in the rows of shrunken patches contain fewer immunostained neurons per 

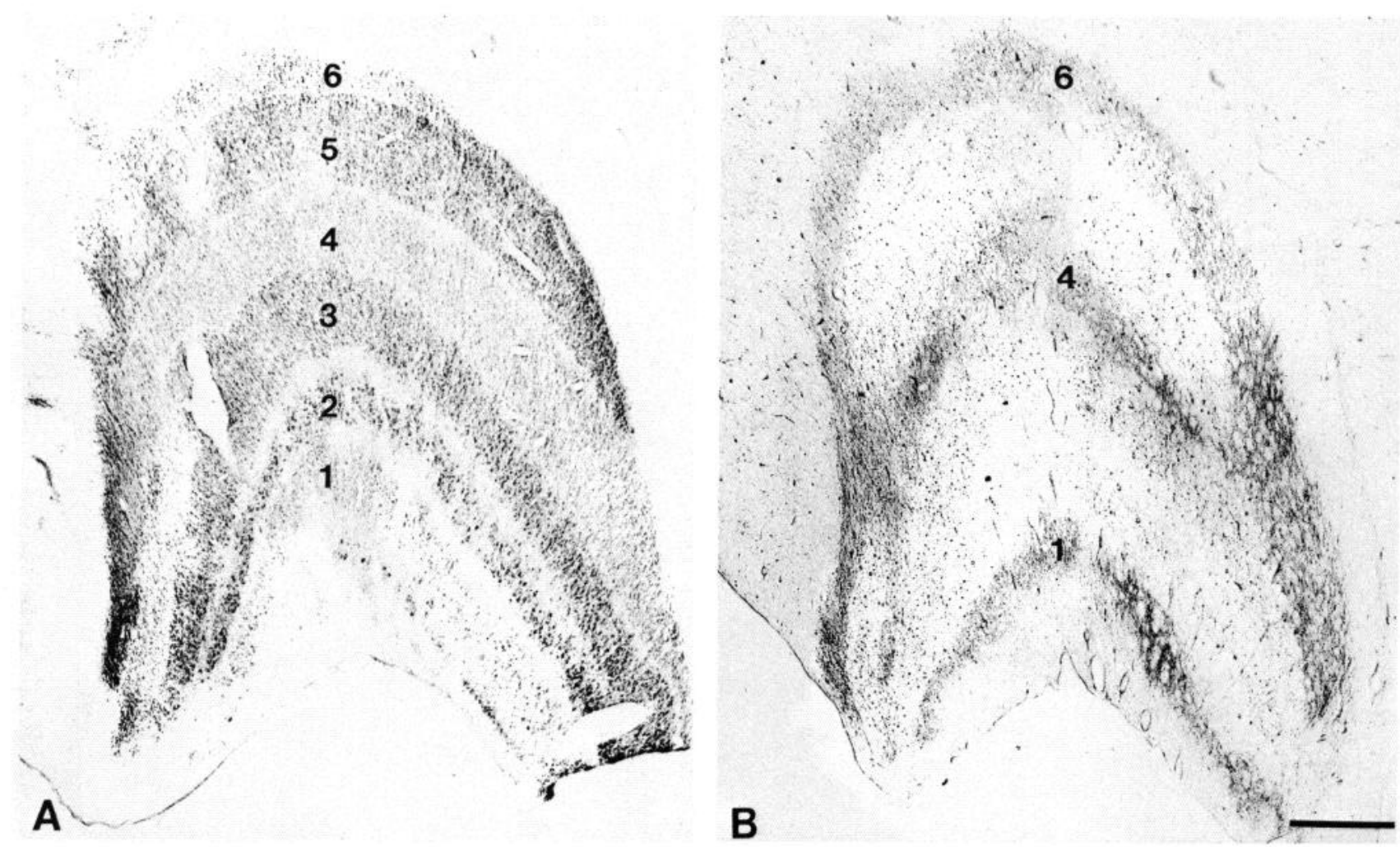

Figure 5. Photomicrographs of sections through the DLG of a monkey in which TTX and HRP were injected in the contralateral eye. This monkey was injected with TTX for $10 \mathrm{~d}$. $A$, Intense $C O$ staining is seen in layers 2,3 , and 5 , while the remaining layers $(1,4$, and 6$)$ are pale. $B$, In a section posterior to the one shown in $A$, retinogeniculate axons and terminals, labeled by the anterograde transport of HRP, occupy layers 1 , 4 and 6 . Bar, $100 \mu \mathrm{m}$.

unit area than do the inter-patch regions of normal monkeys $(t=5.9, p<0.005$; Fig. 3), and the size of these inter-patch regions in the rows of shrunken patches is greater than normal. The inter-row regions of the TTX-injected monkeys also contain significantly lower concentrations of tachykinin-immunoreactive somata than do those of normal monkeys; these reductions occur for inter-row regions in the normal-eye $(t=7.2, p<$ $0.001)$, as well as the injected-eye, columns $(t=10.4, p<0.001)$.

\section{Monocularly aphakic monkeys}

Following the removal of the crystalline lens from one eye of an adult monkey, changes in the $\mathrm{CO}$ staining can be seen in area 17 (Wong et al., 1986). After 3 or 6 months of postoperative survival, striking changes are apparent, principally in layers IIIII, IVB, and V-VI, and the changes affect both sets of COstained rows of patches. The $\mathrm{CO}$ patches in every other row are shrunken, seldom exceed $80 \mu \mathrm{m}$ in diameter, and thus resemble the rows of patches dominated by TTX-injected eyes. By contrast, the patches in the intervening rows are more darkly stained and are enlarged so that they incorporate the inter-patch regions within the rows (Fig. $8 B$ ). Thus, the $\mathrm{CO}$ staining in layers IIIII, IVB, and V-VI consists of elongated rows, homogeneously and darkly stained, that alternate with rows made up of patches that are smaller and more widely separated than normal (Fig. $8 B$ ). In the rows of expanded patches, the increase in the size of the patches occurs both along their lengths (parallel to the long axis of the rows) and widths (perpendicular to the long axis). The mean widths of the elongated patches in the aphakic
Table 2. Number of tachykinin-immunoreactive somata in $3 \mathbf{~ m m} \times$ $100 \mu \mathrm{m}$ rows through layers II and III

\begin{tabular}{llcc} 
Type of row & & Range & Mean \pm SD \\
\hline Normal & $(1)$ & $104-142$ & $130.3 \pm 12.1$ \\
& $(2)$ & $112-148$ & $126.3 \pm 11.0$ \\
TTX (normal) & $(1)$ & $102-151$ & $129.3 \pm 14.9$ \\
& $(2)$ & $109-144$ & $128.1 \pm 16.2$ \\
TTX (shrunken) & $(3)$ & $111-157$ & $133.8 \pm 15.2$ \\
& $(1)$ & $78-99$ & $80.7 \pm 14.2$ \\
& $(2)$ & $82-116$ & $92.3 \pm 10.5$ \\
Aphakic (expanded) & $(3)$ & $77-106$ & $89.5 \pm 11.3$ \\
& $(1)$ & $130-181$ & $157.4 \pm 16.3$ \\
& $(2)$ & $145-190$ & $170.3 \pm 14.4$ \\
& $(3)$ & $143-188$ & $159.0 \pm 11.6$ \\
Aphakic (shrunken) & $(4)$ & $136-181$ & $149.8 \pm 14.1$ \\
& $(1)$ & $38-64$ & $53.8 \pm 9.2$ \\
& $(2)$ & $41-59$ & $49.3 \pm 5.1$ \\
& $(3)$ & $32-70$ & $53.5 \pm 13.2$ \\
& $(4)$ & $44-61$ & $51.4 \pm 5.0$
\end{tabular}

Number of tachykinin-immunoreactive neurons in rows, $3 \mathrm{~mm}$ long $\times 100 \mu \mathrm{m}$ wide, through layers II-III of 2 normal, 3 TTX-injected, and 4 monocularly aphakic monkeys (each line includes the values from a single monkey). The counts of immunostained cells were made by reference to adjacent sections stained with CO. Rows in the TTX-injected monkeys included those stained normally (TTX normal) and those with shrunken patches (TTX shrunken). In monocularly aphakic monkeys, the rows in which patches had fused (Aphakic expanded) and the rows in which the patches had shrunk (Aphakic shrunken) were counted. Values represent counts in 30 rows from each normal monkey, 30 normal and shrunken rows from each TTX-injected monkey, and 30 expanded and shrunken rows from each aphakic monkey. 


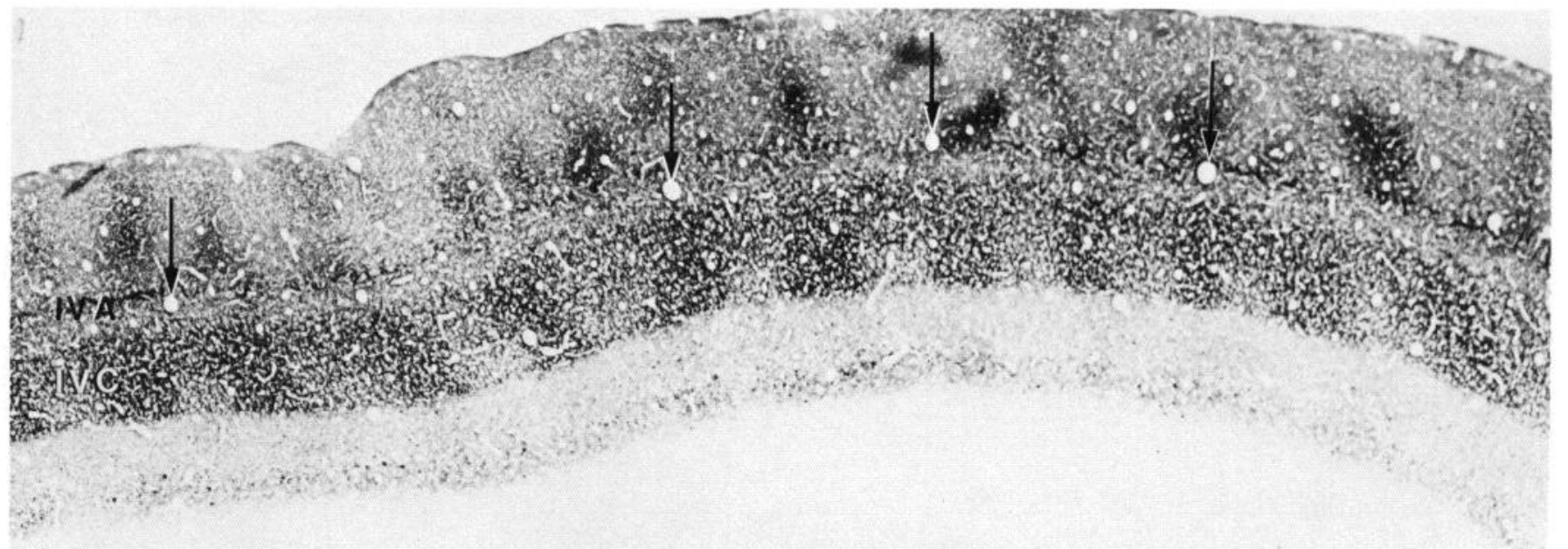

A

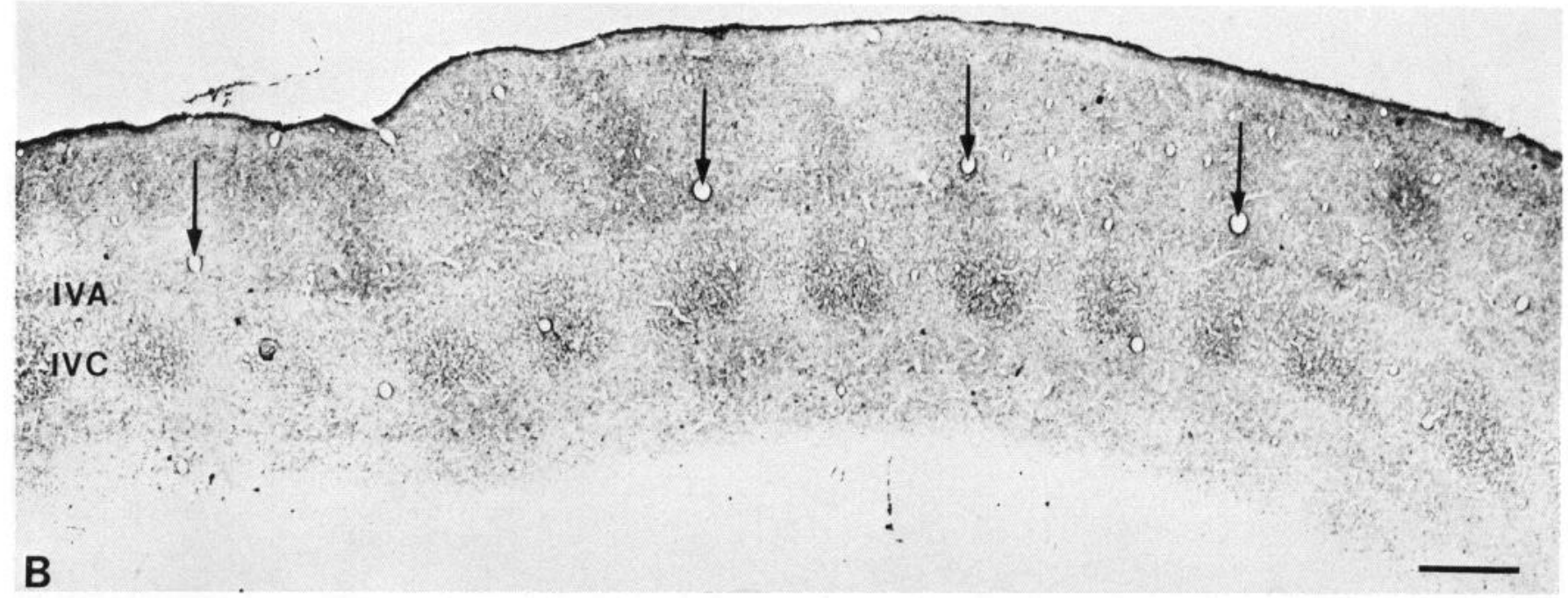

Figure 6. The staining for $\mathrm{CO}(A)$ and for tachykinin-like material $(B)$ in adjacent sagittal sections through area 17 of a monkey injected monocularly with TTX for $15 \mathrm{~d}$. Arrows point to the same blood vessel profiles in the 2 sections. Both the CO staining and the tachykinin immunostaining in layers $I V A$ and $I V C$ are divided into alternating dark and pale regions. The dark $\mathrm{CO}$ regions coincide with the dark immunostained regions, while the pale $\mathrm{CO}$ regions also coincide with pale immunostained regions. Patches in layers II and III are prominent above the dark CO-stained and tachykinin-immunostained regions of layer IV, but are less noticeable above the pale layer IV regions. Bar, $500 \mu \mathrm{m}$.

monkeys are approximately $25 \%$ greater than those in normal monkeys. The changes in area 17 are seen without any change in the CO staining of the LGN and with very modest changes in the staining of layer IVC (Fig. 8C).

Changes comparable to those in the $\mathrm{CO}$ in tachykinin immunostaining of area 17 also occur following monocular lens removal. These changes are clearest in layers II and III, where rows of expanded, immunostained patches alternate with rows of shrunken, immunostained patches (Fig. $8 A$ ). Within the rows of shrunken patches, long stretches (600-800 $\mu \mathrm{m}$ in length) con- taining very few immunostained cell bodies and punctate profiles are interrupted by small patches (approximately 50-100 $\mu \mathrm{m}$ in length) containing relatively large numbers of immunostained cell bodies and puncta. Within the rows of expanded patches, densely packed immunostained cell bodies and profiles, extending as much as $4 \mathrm{~mm}$, are interspersed with narrow (100200- $\mu \mathrm{m}$-wide) gaps containing few immunostained elements (Fig. $8 B$ ). The tachykinin-immunoreactive cell bodies in the layer IIIII rows of aphakic monkeys are the small, relatively lightly stained neurons (see Jones et al., 1988).

Figure 7. Tangential sections through area 17 of a monkey injected monocularly with TTX for $15 \mathrm{~d} . A, B$, Tachykinin immunostaining $(A)$ and CO staining $(B)$ in adjacent sections through the representation of the retinal periphery in area 17. In layers II and III (left one-third of the photomicrographs), the tachykinin and CO staining consist of rows of patches. Every other row contains patches of normal size, while the intervening rows contain patches that are shrunken. The immunostaining and CO staining in layer IVC (right two-thirds of the photomicrograph) consist of alternating dark and pale bands. Comparison of the positions of the same blood vessel profiles (arrowheads) in the 2 sections reveals that the rows of normal tachykinin-stained patches in layers II and III coincide with rows of normal CO-stained patches and that bands of dark tachykinin immunostaining in layer IVC coincide with bands of dark CO staining. Rows of layer II-III patches that are shrunken and layer IVC bands that 

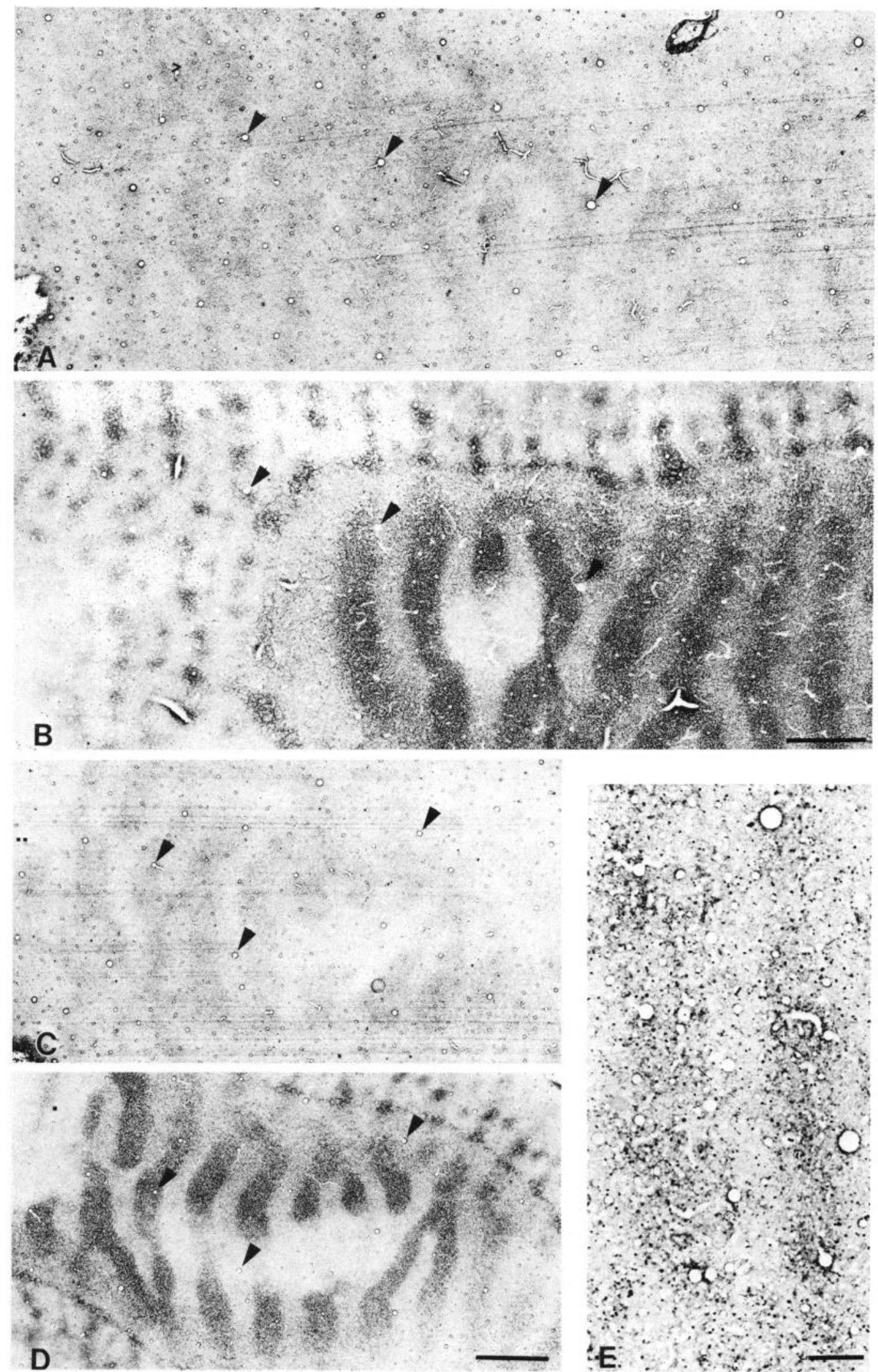

are pale in the tachykinin-immunostained section are also shrunken or pale in the CO-stained section. Bar, $1 \mathrm{~mm}$. $C, D$, Adjacent tangential sections from the foveal representation in area 17 of the same monkey as shown in $A$ and $B$. Complex patterns of interdigitating dark and pale immunostained bands $(C)$ and CO-stained bands $(D)$ are present in layer IVC. Comparison of the positions of the same blood vessels in the 2 sections shows that the dark immunostained bands coincide with dark CO-stained bands, while pale immunostained bands coincide with pale CO-stained bands. Bar, $2 \mathrm{~mm}$. E. Higher-magnification photomicrograph of dark and pale tachykinin-immunostained bands in layer IVC of the same monkey shown in $A-D$. The dark bands contain a greater density of immunostained somata and punctate profiles. Bar, $500 \mu \mathrm{m}$. 
Figure 8. Tangential sections through area 17 of an adult monkey that was killed 3 months following the removal of the crystalline lens from one eye. $A$, The CO staining in layers II and III consists of rows of elongated patches that alternate with rows of shrunken patches. Intense staining fills in the regions between the elongated patches so that some rows are uniformly and darkly stained over lengths of several millimeters. $B$, The section adjacent to $A$, stained immunocytochemically for tachykinin-like substances. In layers II and III, the immunostaining is made up of rows of elongated dark patches, containing high densities of tachykininimmunoreactive somata and punctate profiles, that alternate with pale rows containing shrunken patches of immunoreactive somata and punctate profiles. Comparison of the positions of the same blood vessel profiles (arrowheads) in $A$ and $B$ reveals that rows of elongated $\mathrm{CO}$-stained patches coincide with rows of elongated tachykinin-immunostained patches, while rows of shrunken $\mathrm{CO}$ patches coincide with rows of shrunken immunostained patches. $C$, CO-stained section from the same block as $A$ and $B$. Unlike the $\mathrm{CO}$ staining in layers II and III, the staining in layer $I V C$ closely resembles that of a normal monkey. The only difference from normal CO staining in layer IVC is the presence of thin, intensely stained bands that are visible in some parts of the layer. Tachykinin immunostaining appeared normal in this layer. Rows of elongated $\mathrm{CO}$-stained patches also alternate with rows of shrunken patches in layer $V$. Bar, $1.5 \mathrm{~mm}$.
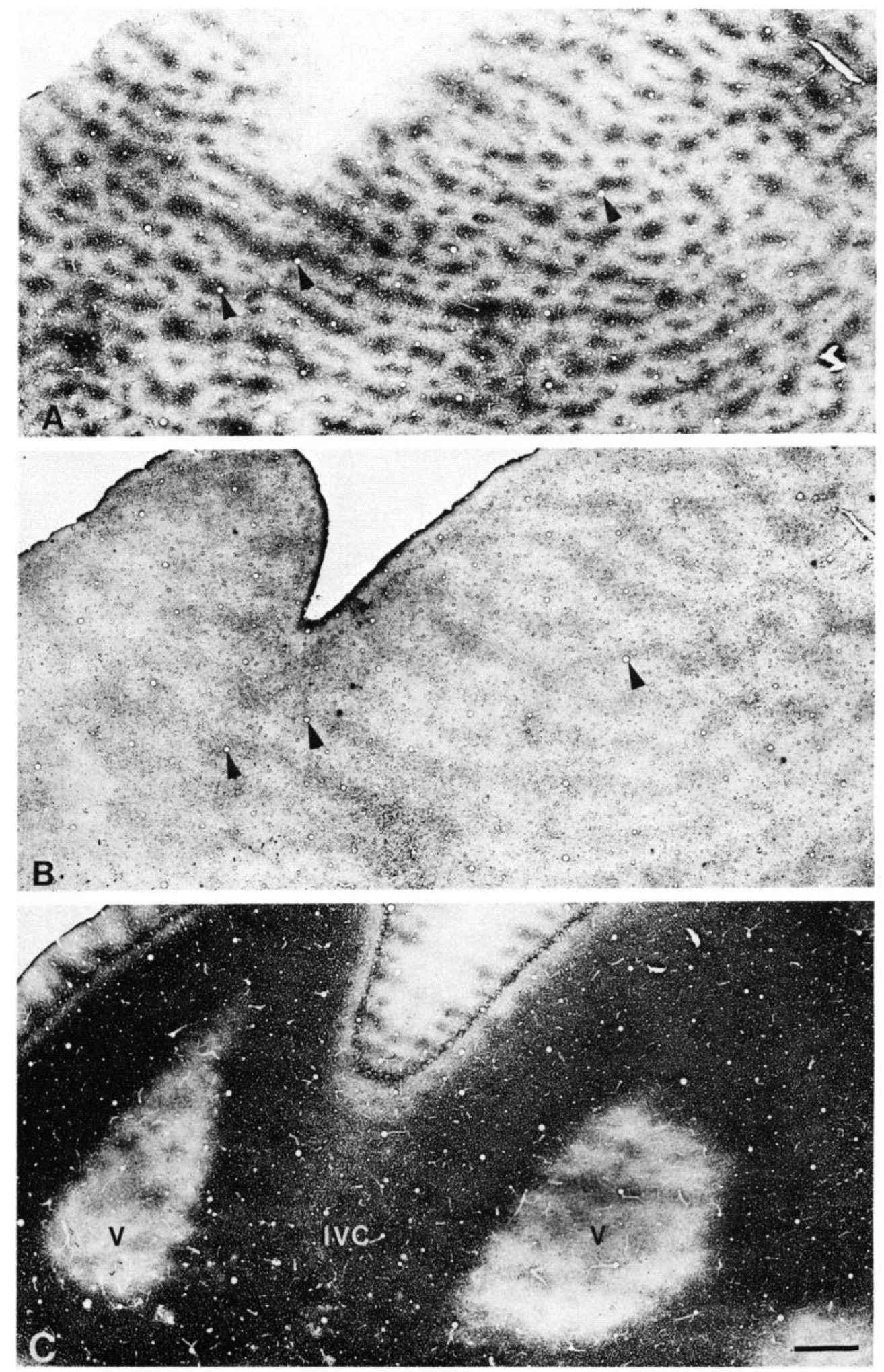

By superimposing the positions of profiles of the same radially oriented blood vessels in adjacent sections through layers IIIII, the CO staining was compared with the tachykinin immunostaining (Fig. 8). The rows of expanded tachykinin-stained patches coincide precisely with the rows of expanded $\mathrm{CO}$ patches, and the rows of shrunken CO-stained patches coincide with the rows of shrunken tachykinin-stained patches.
The differences between normal and aphakic monkeys in the immunostaining of layers II-III were quantified. In rows of expanded patches and in rows of shrunken patches, the numerical densities of immunostained somata differ significantly from normal rows (Table 2). The number of immunostained cells within the rows of elongated patches is significantly greater (approximately $30 \%$ ) than the number within rows from normal 
monkeys $(t-7.0, p<0.001)$, while the number within the rows of shrunken patches is significantly smaller (approximately $60 \%$ less) than the number in normal monkeys ( $t=44.7, p<0.0005$; Table 2). The increased number of tachykinin-immunoreactive somata along a row of expanded patches is accompanied by a significant increase in the numerical density of immunostained cells within individual expanded patches as compared with normal patches $(t-5.8, p<0.001$; Fig. 3$)$. However, the number of immunostained somata per unit area in individual shrunken patches of the aphakic monkeys does not differ significantly from the number in patches of normal monkeys (Fig. 3). As in TTXinjected monkeys, the difference between rows of shrunken patches and rows of normal patches appears to arise from changes in the numerical densities of immunostained somata in regions between patches (Fig. 3) and from changes in the sizes of the interpatch regions.

Little change is found in CO staining of layer IVC in aphakic monkeys, with only thin (approximately $120-\mu \mathrm{m}$-wide) bands of slightly darker staining interrupting the usual dark, homogeneous staining (Fig. 8C). Within layer IVC of these monkeys, the tachykinin immunostaining appears homogeneous. None of the other layers (IVB, V, and VI) displaying changes in $\mathrm{CO}$ staining contains significant populations of the small tachykininpositivc somata or punctate profiles and, thus, none exhibits any detectable changes in tachykinin immunostaining. No changes were seen the numbers of the large, intensely immunoreactive cells (Jones et al., 1988) in any layer.

\section{Discussion}

In the present study we found that the immunostaining for a tachykinin-like substance in neurons of the monkey visual cortex is regulated by activity. Manipulations of one eye that reduce staining for the mitochondrial enzyme $\mathrm{CO}$ in the visual cortex lead to marked reductions in the number of tachykinin-immunoreactive somata and terminal-like punctate profiles, while manipulations that increase $\mathrm{CO}$ staining increase the number of immunostained elements. The results of the preceding study (Jones et al., 1988) suggest that the tachykinin-like substances exhibiting these changes include SP, SK, and possibly one or more other members of the tachykinin family. We used the relative intensity of $\mathrm{CO}$ staining as an indicator of metabolic activity in monkey area 17 . Since CO staining appears to be an accurate marker for chronic patterns of metabolic activity in area 17 (Horton and Hubel, 1981; Humphrey and Hendrickson, 1983 ) and is localized preferentially to neuronal mitochondria in this area (Carroll and Wong-Riley, 1984), we interpret the changes in staining produced by long-term eyc manipulations as indicating changes in neuronal activity.

\section{Activity-dependent regulation of immunostaining}

Reductions in CO staining were seen in area 17 of both TTXinjected (see also Wong-Riley and Carroll, 1984) and aphakic monkeys. In each case the reduction in histochemical staining or in the area occupied by the CO patches in layers II-III was accompanied by reductions in the number of tachykinin-immunoreactive neurons (Fig. 9). Since these reductions occur without a change in the total number of thionin-stained neurons, the decline in immunostained numbers is most likely due to mechanisms other than cell death. The results suggest that deprivation reduces the concentration of tachykinin-like material within individual neurons, so as to leave them undetectable by immunocytochemical methods. Similar findings and interpre- tations have been reported previously for monkey cortical neurons displaying GABA- and GAD-like immunoreactivities (Hendry and Jones, 1986).

Intraocular injections of TTX reduce the numerical density of tachykinin-immunoreactive cells within layers II and III of both ocular dominance columns (throughout the injected-eye columns and in the inter-row regions of the uninjected-eye columns). By contrast, tachykinin immunostaining in layer IVC is reduced only in the injected-eye columns. This difference in TTX effects most likely occurs because the neurons of layer IVC are monocularly driven (e.g., Hubel and Wiesel, 1968), while the neurons between rows of $\mathrm{CO}$ patches are normally driven by both eyes (Livingstone and Hubel, 1984). The loss of input from one eye may be responsible for the reductions in immunocytochemical staining within these binocularly driven neurons.

No damage was observed when the retinas of both the injected and normal eyes were examined histologically. The transport of HRP and tritiated proteins to the LGN also indicates that retinal ganglion cells continued to be viable following the last TTX injection, and suggests that the dosages of TTX we used did not greatly inhibit the anterograde transport of macromolecules. These results indicate that the effects of the TTX injections can be directly attributed to the reduction in neuronal activity along the visual pathway and suggest that the reductions in tachykinin immunostaining seen in area 17 are activity-dependent.

The removal of the crystalline lens from one eye of an adult monkey causes the CO-stained patches in every other row to shrink and the patches in the alternating rows to expand. It might be assumed that the shrunken patches are related to the aphakic eye because of their similarity to the shrunken patches dominated by a TTX-injected eye. However, since both sets of alternating rows of patches are affected by monocular aphakia, the determination of which row is related to the aphakic eye and which to the normal eye will require additional experiments. In normal monkeys, the neurons in the $\mathrm{CO}$ patches differ functionally from the neurons outside the patches, particularly in their selectivity for the orientation, color, and spatial frequency of a visual stimulus (Humphrey and Hendrickson, 1983; Tootell et al., 1983; Livingstone and Hubel, 1984). Removal of the crystalline lens affects the perception of color (Spitalny et al., 1969) and eliminates responses to visual stimuli of high spatial frequencies (von Noorden and Crawford, 1977; von Noorden, 1985). These changes, originating at the visual periphery, may lead to the changes in cortical $\mathrm{CO}$ staining. A fuller treatment of the effects of aphakia on the CO staining in adult monkey area 17 will be presented elsewhere, but the principal point we wish to make here is that the expansion and fusion of $\mathrm{CO}$ patches within every other row are likely to represent a protracted increase in the metabolic activity of neurons between and immediately around the patches.

With the apparent increase in metabolic activity between and around $\mathrm{CO}$ patches in every other row of aphakic monkey area 17 , the density of tachykinin-immunoreactive neurons increases and the territory occupied by dense clusters of immunostained puncta expands. Such increases in the numbers of immunostained cell bodies and puncta could result from either the induction of tachykinin expression within neurons that previously did not express these peptides or the increase in tachykinin synthesis within neurons that previously synthesized or retained too little peptide to be detected immunocytochemically. There 
NORMAL

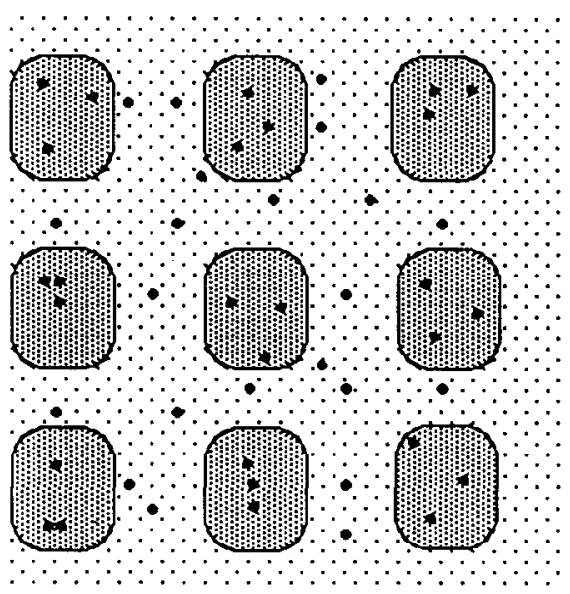

APHAKIC

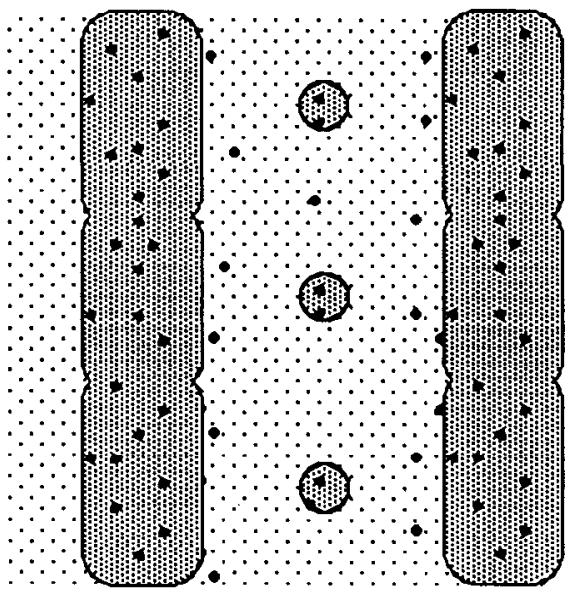

Figure 9. Schematic diagram of tachykinin immunostaining in layers IIIII of monkey area 17. In normal monkeys, immunoreactive cell bodies (large circles) are evenly distributed, but stained puncta (small dots) are densely clustered in periodic patches that line up in rows. In aphakic monkeys, all rows of immunostained patches are affected. Patches in every other row expand and the density of stained cell bodies increases; patches in alternating rows shrink and the density of cell bodies declines. In TTX-injected monkeys, the patches in every other row remain normal, with reductions in cell bodies seen only around the patches, and the patches in the alternating rows shrink.

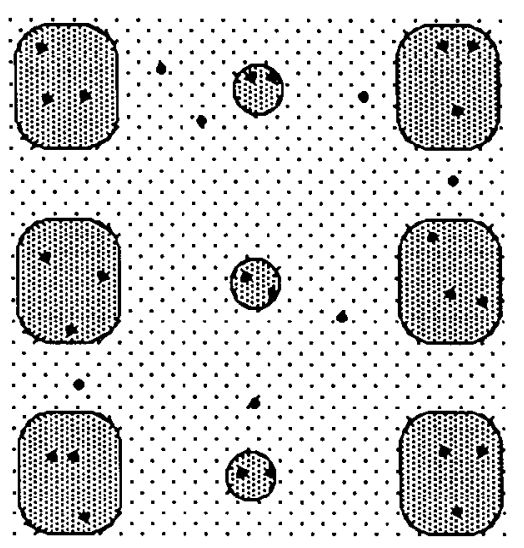

cortex (Jones et al., 1988). Comparisons of the present findings with the findings of previous studies (Hendry et al., 1987) indicate that the numerical density of the tachykinin-immunoreactive cells is approximately half that of GABA-immunoreactive cells in layer IVC. Thus, 2 equal populations of GABA cells appear to exist in layer IVC: those with tachykinin-like immunoreactivity and those without. Colocalization studies of layer IVC neurons support this suggestion (Jones et al., 1988; S. H. C. Hendry and E. G. Jones, unpublished observations). The 2 populations may be differentially sensitive to monocular deprivation or enucleation. In deprived-eye columns, the number of GABA-immunoreactive cells through layer IVC declines by one-half (Hendry and Jones, 1986), while the number of tachykinin-immunoreactive cells in such columns is often reduced to 0 . These data might indicate that GABA and tachykinin immunostaining is reduced in the same population of layer IVC cells and that the GABA cells remaining after deprivation are those containing no tachykinin-like substances; i.e., the GABA neurons most affected by visual deprivation may be the neurons in which tachykinin-like immunoreactivity coexists. Alternatively, the effect of deprivation on tachykinin immu- 
nostaining may be independent of the effect on GABA immunostaining, so that the GABA cells remaining may include some that normally express tachykinins and some that do not.

GABA- and tachykinin-like immunoreactivities also coexist within neurons of layers II-III (Jones et al., 1988). Like the total population of GABA cell bodies in layers II-III of normal monkeys (Hendrickson et al., 1981; Fitzpatrick et al., 1983; Hendry et al., 1987), the GABA subpopulation expressing tachykininlike immunoreactivity bears no consistent spatial relationship to the $\mathrm{CO}$ patches of these layers. The findings of the present study suggest that, in aphakic monkeys, the increases in $\mathrm{CO}$ staining are accompanied by increases in the number of tachykinin-immunoreactive cells within the $\mathrm{CO}$ patches and in the regions between them. Preliminary colocalization studies on aphakic monkeys indicate that these cells are also GABA-immunoreactive and morphologically identical to the ones within the normal $\mathrm{CO}$ patches. Thus, a type of GABA cell diffusely distributed within layers II and III appears capable of synthesizing relatively high concentrations of tachykinins under normal circumstances. However, a larger population of GABA cells may retain the capacity for tachykinin synthesis and, under circumstances of greater metabolic activity, these cells contain sufficient tachykinin-like material to be stained immunocytochemically.

Other populations of GABA neurons in monkey cerebral cortex are immunoreactive for the neuropeptides somatostatin, neuropeptide $\mathrm{Y}$ or cholecystokinin octapeptide (Hendry et al., 1984a). The immunostaining for these peptides might also be affected by changes in metabolic activity, but in area 17 they are present within neurons and processes outside the layers that not only receive direct input from the LGN (Hendry et al., 1984b; Campbell et al., 1987), but that also show the most overt changes with monocular deprivation in adults (Hendry and Jones, 1986; Hendry and Kennedy, 1986; and the present study).

\section{Functional implications}

Although few reports of functional plasticity in adult monkey area 17 have been published, there are indications that the responsiveness to visual stimulation in one eye can be altered in adulthood (LcVay ct al., 1980). Such changes in neuronal physiology might result, in part, from fluctuations in the levels of neuroactive substances within cortical cells and axons. That is, changes in the concentration of tachykinins within area 17 neurons may lead to identifiable changes in the functional properties of these cells, or, more likely, of their targets. At least one tachykinin, SP, is neuroactive when applied iontophoretically onto neurons of the mammalian cerebral cortex (Lamour et al., 1983; Jones and Olpe, 1984). However, little is known of the normal actions of SP and of other tachykinins in the cerebral cortex, and, thus, the effects produced by changes in the peptides levels within cortical neurons are unclear.

Both the eye manipulations used in this study and the cortical effects involved large populations of neurons. A major question concerns the changes in neurotransmitter expression that may accompany more subtle changes in visual input: Is the synthesis of cortical neuroactive substances altered significantly by changes in the daily visual environment? We used monocular deprivation because the limits of the detectability of immunocytochemical changes dictated that large, spatially segregated populations of neurons should be affected for the changes to be obvious. To determine whether rapid shifts in the synthesis or effective concentration of neurotransmitters are a normal part of cortical function, more direct measures of synthesis, such as the chemical activity of anabolic enzymes or the abundance of messenger RNAs encoding for an enzyme or peptide, will have to be used on single neurons.

\section{References}

Baker, H., T. Kawano, F. L. Margolis, and T. H. Joh (1983) Transneuronal regulation of tyrosine hydroxylase expression in olfactory bulb of mouse and rat. J. Neurosci. 3: 69-78.

Black, I. B., J. E. Adler, C. F. Dreyfus, G. M. Jonakait, D. M. Katz, E. F. LaGamma, and K. M. Markey (1984) Neurotransmitter plasticity at the molecular level. Science 225: 1266-1270.

Blakemore, C., L. J. Garey, and F. Vital-Durand (1978) The physiological effects of monocular deprivation and their reversal in the monkey's cortex. J. Physiol. (Lond.) 261: 423-444.

Campbell, M. J., D. A. Lewis, R. Benoit, and J. H. Morrison (1987) Regional heterogeneity in the distribution of somatostatin-28- and somatostatin-28 $1-12$-immunoreactive profiles in monkey neocortex. $\mathbf{J}$. Neurosci. 7: 1133-1144.

Carroll, E. W., and M. T. T. Wong-Riley (1984) Quantitative light and electron microscopic analysis of cytochrome oxidase-rich zones in the striate cortex of the squirrel monkey. J. Comp. Neurol. 221. $1-17$.

DeOlmos, J., H. Hardy, and L. Heimer (1978) The afferent connections of the main and accessory olfactory bulb formations in the rat: An experimental HRP study. J. Comp. Neurol. 181: 213-241.

Fitzpatrick, D., J. S. Lund, and D. Schmechel (1983) Glutamic acid decarboxylase immunoreactive neurons and terminals in the visual cortex of monkey and cat. Soc. Neurosci. Abstr. 9: 616.

Hendrickson, A. E., S. P. Hunt, and J.-Y. Wu (1981) Immunocytochemical localization of glutamic acid decarboxylase in monkey striate cortex. Nature 292: 605-607.

Hendry, S. H. C., and E. G. Jones (1986) Reduction in number of immunostained GABAergic neurons in deprived-eye dominance columns of monkey area 17 . Naturc 320:750-753.

Hendry, S. H. C., and M. B. Kennedy (1986) Immunoreactivity for a calmodulin-dependent protein kinase is selectively increased in macaque striate cortex after monocular deprivation. Proc. Natl Acad. Sci. USA 83: 1536-1540.

Hendry, S. H. C., E. G. Jones, J. DeFelipe, D. E. Schmechel, C. Brandon, and P. C. Emson (1984a) Neuropeptide-containing neurons of the cerebral cortex are also GABAergic. Proc. Natl. Acad. Sci. USA 81: 6526-6530.

Hendry, S. H. C., E. G. Jones, and P. C. Emson (1984b) Morphology, distribution and synaptic relations of somatostatin- and neuropeptide $\mathrm{Y}$-immunoreactive neurons in rat and monkey cerebral cortex. $\mathbf{J}$. Neurosci. 4: 2497-2517.

Hendry, S. H. C., H. D. Schwark, E. G. Jones, and J. Yan (1987b) Numbers and proportions of GABA immunoreactive neurons in different areas of monkey cerebral cortex. J. Neurosci. 7: 1503-1519.

Horton, J. C., and D. H. Hubel (1981) Regular patchy distribution of cytochrome oxidase staining in primary visual cortex of macaque monkey. Nature 292: 762-764.

Hubel, D. H., and T. N. Wiesel (1968) Receptive fields and functional architecture of monkey striate cortex. J. Physiol. (Lond.) 195: 215243.

Hubel, D. H., T. N. Wiesel, and S. LeVay (1977) Plasticity of ocular dominance columns in monkey striate cortex. Phil. Trans. R. Soc. Lond. [Biol.] 278: 377-409.

Humphrey, A. I.., and A. E. Hendrickson (1983) Background and stimulus-induced patterns of high metabolic activity in the visual cortex (area 17) of the squirrel and macaque monkey. J. Neurosci. 3: 345-358.

Jones, E. G., J. D. Coulter, and S. P. Wise (1979) Commissural columns in the sensory-motor cortex of monkeys. J. Comp. Neurol. 188: 113-135.

Jones, E. G., J. DeFelipe, S. H. C. Hendry, and J. E. Maggio (1988) A study of tachykinin-immunoreactive neurons in monkey cerebral cortex. J. Neurosci. 8: 1206-1 224.

Jones, R. S. G., and H.-R. Olpe (1984) Activation of the noradrenergic projection from locus coeruleus reduces the excitatory responses of anterior cingulate cortical neurones to substance P. Neuroscience 13: $819-825$ 
Kantner, R. M., M. L. Kirby, and B. D. Goldstein (1985) Increase in substance $\mathrm{P}$ in the dorsal horn during a chemogenic stimulus. Brain Res. 338: 196-199.

LaMotte, C., C. B. Pert, and S. H. Snyder (1976) Opiate receptor binding in primate spinal cord: Distribution and changes after dorsal root section. Brain Res. 112: 407-412.

Lamour, Y., P. Dutar, and A. Jobert (1983) Effects of neuropeptides on rat cortical ncurons: Laminar distribution and interaction with the effect of acetylcholine. Neuroscience 10: 107-117.

LeVay, S., T. N. Wiesel, and D. H. Hubel (1980) The development of ocular dominance columns in normal and visually deprived monkeys. J. Comp. Neurol. 191: 1-51.

Livingstone, M. S., and D. H. Hubel (1984) Anatomy and physiology of a color system in the primate visual cortex. J. Neurosci. 4: 309356.

Molinoff, P. B., S. Brimijoin, R. Weinshilboum, and J. Axelrod (1970) Neurally mediated increase in dopamine- $\beta$-hydroxylase activity. Proc. Natl. Acad. Sci. USA 66: 453-457.

Ninkovic, M., S. P. Hunt, and J. S. Kelly (1981) Effect of dorsal rhizotomy on the autoradiographic distribution of opiate and neurotensin receptors and neurotensin-like immunoreactivity within the rat spinal cord. Brain Res. 230: 111-119.
Spitalny, L. A., J. B. Devoe, and H. D. Fenske (1969) Color perception in unilateral aphakia. Arch. Ophthalmol. 82: 592-595.

Stryker, M. P., and W. A. Harris (1986) Binocular impulse blockade prevents the formation of ocular dominance columns in cat visual cortex. J. Neurosci. 6: 2117-2133.

Tootell, R. B. H., M. S. Silverman, R. L. DeValois, and G. H. Jacobs (1983) Functional organization of the second cortical visual area in primates. Science 220: 737-739.

von Noorden, G. K. (1985) Amblyopia: A multidisciplinary approach. Invest. Ophthalmol. Vis. Sci. 26: 1704-1716.

von Noorden, G. K., and M. L. J. Crawford (1977) Form deprivation without light deprivation produces the visual deprivation syndrome in Macaca mulatta. Brain Res. 129: 37-51.

Wong, E., S. H.C. Hendry, E. G. Jones, and N. Burstein (1986) Cortical metabolic changes produced by lens removal in adult monkeys. Invest. Ophthalmol. Vis. Sci. 27: 76.

Wong-Riley, M. T. T. (1979) Changes in the visual system of monocularly sutured or enucleated kittens demonstrable with cytochrome oxidase histochemistry. Brain Res. 171: 11-28.

Wong-Riley, M., and E. W. Carroll (1984) Effect of impulse blockade on cytochrome oxidase activity in monkey visual system. Nature 307: 262-264. 